INPLASY

PROTOCOL

To cite: Clemente et al. A systematic review of accuracy, precision and repeatability of technical and tactical fieldbased tests in soccer. Inplasy protocol 202130101. doi: 10.37766/inplasy2021.3.0101

Received: 27 March 2021

Published: 27 March 2021

Corresponding author:

Filipe Manuel Clemente

filipe.clemente5@gmail.com

Author Affiliation:

Escola Superior Desporto e Lazer, Instituto Politécnico de Viana do Castelo, Portugal

Support: None.

Review Stage at time of this submission: Preliminary searches.

Conflicts of interest: None declared.

\section{A systematic review of accuracy, precision and repeatability of technical and tactical field-based tests in soccer}

Clemente, FM¹; Praça, G²; Akyildiz, Z3; Afonso, J4.

Review question / Objective: This systematic review was conducted to: (1) summarize the accuracy, precision, and repeatability levels of technical and tactical field-based tests applied in soccer; and (2) provide a list of the precise and reliable technical and tactical field-based tests to different age-groups and sexes.

Condition being studied: Soccer players exposed to technical and/or tactical field-based tests covering the: (i) individual technical skills (e.g., pass, dribble); (ii) tactical behavior; (iii) tactical knowledge; (iv) decision-making in the field.

Information sources: Electronic databases (EBSCO, PubMed, Scielo, SPORTDiscus, Web of Science) were searched for relevant publications on April 06, 2021. Additionally, the reference lists of the studies retrieved were manually searched to identify potentially eligible studies not captured by the electronic searches. Finally, an external expert has been contacted in order to verify the final list of references included in this scoping review in order to understand if there was any study that was not detected through our research.

INPLASY registration number: This protocol was registered with the International Platform of Registered Systematic Review and Meta-Analysis Protocols (INPLASY) on 27 March 2021 and was last updated on 27 March 2021 (registration number INPLASY202130101).

\section{INTRODUCTION}

Review question / Objective: This systematic review was conducted to: (1) summarize the accuracy, precision, and repeatability levels of technical and tactical field-based tests applied in soccer; and (2) provide a list of the precise and reliable technical and tactical field-based tests to different age-groups and sexes.

Rationale: Technical and tactical dimensions are determinants in soccer. Therefore, a proper assessment of the 
technical level and tactical behavior of players is necessary to adjust the training process. However, assessing the accuracy, precision, and repeatability of instruments is of paramount importance to reduce the possible erroneous interpretations.

Condition being studied: Soccer players exposed to technical and/or tactical fieldbased tests covering the: (i) individual technical skills (e.g., pass, dribble); (ii) tactical behavior; (iii) tactical knowledge; (iv) decision-making in the field.

\section{METHODS}

Search strategy: Electronic databases (EBSCO, PubMed, Scielo, SPORTDiscus, Web of Science) were searched for relevant publications on April 6, 2021. Keywords and synonyms were entered in various combinations: [(soccer OR football) AND ("Validity" OR "Accuracy" OR "Reliability" OR "Precision" OR "Variability" OR "Repeatability" OR "Reproducibility" OR "Consistency")] AND one code line for MeSH terms ("techni"” OR "tactic*" OR "skill*" OR "decision*" OR "psychomotor"). Additionally, the reference lists of the studies retrieved were manually searched to identify potentially eligible studies not captured by the electronic searches. Finally, an external expert has been contacted in order to verify the final list of references included in this scoping review in order to understand if there was any study that was not detected through our research.

Participant or population: Soccer players from any age group, competitive level or sex.

Intervention: Exposed to technical and/or tactical field-based tests covering the: (i) individual technical skills (e.g., pass, dribble); (ii) tactical behavior; (iii) tactical knowledge; (iv) decision-making in the field.

Comparator: A laboratorial test and/or a concurrent field-based test.
Study designs to be included: No restrictions with regard to study design. In case of more than one time-point, both (pre and post) will be considered.

Eligibility criteria: Inclusion criteria: (i) Soccer players from any age group, competitive level or sex; (ii) Exposed to technical and/or tactical field-based tests covering the: (i) individual technical skills (e.g., pass, dribble); (ii) tactical behavior; (iii) tactical knowledge; (iv) decision-making in the field; (iii) A laboratorial test and/or a concurrent field-based test; (iv) Measures of accuracy/precision (e.g., typical error; mean absolute error) and/or repeatability (e.g., intraclass correlation test; coefficient of variation); (v) No restrictions with regard to study design. In case of more than one time-point, both (pre and post) will be considered; (vi) Only original and full-text studies written in English, Portuguese, Spanish, Italian and French. Exclusion criteria: (i) Sports other than soccer (e.g., futsal, beach football, basketball, handball), physical education students, general population; (ii) Physical tests (e.g., aerobic performance, anaerobic performance); laboratory-based tests (e.g., room, simulated games, watching videos); (iii) No laboratorial tests and/or concurrent technical/tactical field-based tests; (iv) No statistics related to accuracy/precision and/or repeatability; (v) Written in languages other than English, Portuguese, Spanish, Italian and French. Other article types than original (e.g., reviews, letters to editors, trial registrations, proposals for protocols, editorials, book chapters and conference abstracts).

Information sources: Electronic databases (EBSCO, PubMed, Scielo, SPORTDiscus, Web of Science) were searched for relevant publications on April 06, 2021. Keywords and synonyms were entered in various combinations: [(soccer OR football) AND ("Validity" OR "Accuracy" OR "Reliability" OR "Precision" OR "Variability" OR "Repeatability" OR "Reproducibility" OR "Consistency")] AND one code line for MeSH terms ("techni") OR "tactic*" OR "skill*" OR "decision*" OR "psychomotor"). Additionally, the reference lists of the 
studies retrieved were manually searched to identify potentially eligible studies not captured by the electronic searches. Finally, an external expert has been contacted in order to verify the final list of references included in this scoping review in order to understand if there was any study that was not detected through our research.

Main outcome(s): Measures of accuracy/ precision (e.g., typical error; mean absolute error) and/or repeatability (e.g., intraclass correlation test; coefficient of variation).

Quality assessment / Risk of bias analysis: The Downs \& Black methodological assessment will be used.

Strategy of data synthesis: The following information was extracted from the included original articles: (i) validity measure (e.g., typical error, absolute mean error); and (ii) reliability measure (e.g., intraclass correlation test, coefficient of variation). Additionally, the following data items were extracted: (i) number of participants (n), age-group (youth, adults or both), sex (men, women or both), training level (untrained, trained); (ii) characteristics of the experimental approach to the problem, procedures and settings of each study and the details of the tests and implementation. Additionally, information about ecological validity, if available, will be described.

Subgroup analysis: None.

Sensitivity analysis: None.

Language: English.

Country(ies) involved: Portugal; Brazil; Turkey.

Keywords: football; technical skill; tactical behavior; agility.

Contributions of each author:

Author 1 - Filipe Manuel Clemente - Lead the project, wrote and revised the original manuscript.
Author 2 - Gibson Praça - Wrote and revised the original manuscript.

Author 3 - Zeki Akyildiz - Wrote and revised the original manuscript.

Author 4 - José Afonso - Run the data search, performed the methodological assessment, conducted the data extraction, wrote and revised the original manuscript. 